\title{
Preparation and Reactivity of Mixed-ligand Ruthenium(II) Hydride Complexes with Phosphites and Polypyridyls
}

\author{
Gabriele Albertin,* Stefano Antoniutti, Alessia Bacchi, Claudia D'Este, and Giancarlo \\ Pelizzi
}

SUPPORTING INFORMATION

\section{EXPERIMENTAL DETAILS AND SPECTROSCOPIC DATA (IR, NMR)}

$\left[\mathrm{RuH}(\mathrm{bpy})\left(\mathrm{PPh}_{2} \mathrm{OEt}\right)_{3}\right] \mathrm{CF}_{3} \mathrm{SO}_{3}\left(\mathbf{4}-\mathrm{CF}_{3} \mathrm{SO}_{3}\right)$. To a solution of $\mathrm{RuCl}_{4}(\mathrm{bpy}) \cdot \mathrm{H}_{2} \mathrm{O}(0.5$ $\mathrm{g}$, about $1.20 \mathrm{mmol})$ in $10 \mathrm{~mL}$ of ethanol were added first an excess of $\mathrm{PPh}_{2} \mathrm{OEt}(6.25 \mathrm{mmol}, 1.44$ $\mathrm{mL})$ and then an excess of $\mathrm{NaBH}_{4}(12.5 \mathrm{mmol}, 0.47 \mathrm{~g})$ in $20 \mathrm{~mL}$ of ethanol. The reaction mixture was stirred at room temperature for $3 \mathrm{~h}$ and then the solvent was removed under reduced pressure. From the oil obtained, the hydride $\left[\mathrm{RuH}(\mathrm{bpy}) \mathrm{P}_{3}\right] \mathrm{Cl}$ was extracted with three $10-\mathrm{mL}$ portions of dichloromethane and the extracts were evaporated to dryness. An excess of $\mathrm{LiCF}_{3} \mathrm{SO}_{3}(5 \mathrm{mmol}$, $0.78 \mathrm{~g}$ ) in $4 \mathrm{~mL}$ of ethanol was added to the oil obtained and the resulting solution was stirred for 1 h. The solvent was removed under reduced pressure giving a red-brown oil, from which the complex was extracted with three $5-\mathrm{mL}$ portions of diethyl ether. By cooling to $-25^{\circ} \mathrm{C}$ of the extracts, red-brown microcrystals of the complex separated out which were filtered and dried under vacuum; yield $\geq 25 \%$. Anal. Calcd for $\mathrm{C}_{53} \mathrm{H}_{54} \mathrm{~F}_{3} \mathrm{~N}_{2} \mathrm{O}_{6} \mathrm{P}_{3} \mathrm{RuS}$ : C, 57.97; H, 4.96; N, 2.55. Found: C, 58.06; H, 4.84; N, 2.45. $\Lambda_{\mathrm{M}}=87.1 \Omega^{-1} \mathrm{~mol}^{-1} \mathrm{~cm}^{2}$. IR (KBr): $1970 \mathrm{w}[v(\mathrm{RuH})] \mathrm{cm}^{-1} .{ }^{1} \mathrm{H}$ NMR $\left[\mathrm{CD}_{2} \mathrm{Cl}_{2}, 25^{\circ} \mathrm{C} ; \delta\right]: 8.54-6.51\left(\mathrm{~m}, 38 \mathrm{H}, \mathrm{Ph}\right.$ and bpy), $3.30 \mathrm{~m}, 2.95$ qnt $\left(6 \mathrm{H}, \mathrm{CH}_{2}\right), 0.60,0.31$ (t, 9 $\left.\mathrm{H}, \mathrm{CH}_{3}\right),-12.98\left(\mathrm{dt}, 1 \mathrm{H}, \mathrm{H}^{-}\right) .{ }^{31} \mathrm{P}\left\{{ }^{1} \mathrm{H}\right\} \mathrm{NMR}\left[\mathrm{CD}_{2} \mathrm{Cl}_{2}, 25{ }^{\circ} \mathrm{C} ; \delta\right]$ : spin system $\mathrm{AB}_{2}, \delta_{\mathrm{A}} 155.8, \delta_{\mathrm{B}}$ $141.5, J_{\mathrm{AB}}=40.0 \mathrm{~Hz}$.

$\left[\mathrm{RuH}(\right.$ phen $\left.)\left(\mathrm{PPh}_{2} \mathrm{OEt}\right)_{3}\right] \mathrm{CF}_{3} \mathrm{SO}_{3}\left(\mathbf{5 C}_{-} \mathrm{CF}_{3} \mathrm{SO}_{3}\right)$. This complex was prepared like the related 2,2'-bipyridine derivative $\mathbf{4} \mathbf{c}-\mathbf{C F}_{3} \mathrm{SO}_{3}$ using $\mathrm{LiCF}_{3} \mathrm{SO}_{3}$ as the precipitating agent; yield 
$\geq 25 \%$. Anal. Calcd for $\mathrm{C}_{55} \mathrm{H}_{54} \mathrm{~F}_{3} \mathrm{~N}_{2} \mathrm{O}_{6} \mathrm{P}_{3} \mathrm{RuS}$ : C, 58.87; H, 4.85; N, 2.50. Found: C, 58.99; H, 4.77; $\mathrm{N}, 2.40 . \Lambda_{\mathrm{M}}=86.6 \Omega^{-1} \mathrm{~mol}^{-1} \mathrm{~cm}^{2}$. IR $(\mathrm{KBr}): 1978 \mathrm{w}[\mathrm{v}(\mathrm{RuH})] \mathrm{cm}^{-1} .{ }^{1} \mathrm{H} \mathrm{NMR}\left[\mathrm{CD}_{2} \mathrm{Cl}_{2}\right.$, $25^{\circ} \mathrm{C} ; \delta$ ]: 9.03-6.19 (m, $38 \mathrm{H}, \mathrm{Ph}$ and phen), $3.42 \mathrm{~m}, 3.05 \mathrm{qnt}\left(6 \mathrm{H}, \mathrm{CH}_{2}\right), 0.61,0.39$ (t, $9 \mathrm{H}, \mathrm{CH}_{3}$ ), $-12.67\left(\mathrm{dt}, 1 \mathrm{H}, \mathrm{H}^{-}\right) .{ }^{31} \mathrm{P}\left\{{ }^{1} \mathrm{H}\right\} \mathrm{NMR}\left[\mathrm{CD}_{2} \mathrm{Cl}_{2}, 25^{\circ} \mathrm{C} ; \delta\right]$ : spin system $\mathrm{AB}_{2}, \delta_{\mathrm{A}} 156.1, \delta_{\mathrm{B}} 141.9$, $J_{\mathrm{AB}}=40.5 \mathrm{~Hz}$.

$\left[\mathrm{RuH}(\mathrm{bpy})_{2} \mathrm{P}\right] C F_{3} \mathrm{SO}_{3}\left(8-\mathrm{CF}_{3} \mathrm{SO}_{3}\right)\left[\mathrm{P}=\mathrm{P}(\mathrm{OEt})_{3}(\mathrm{a}), \operatorname{PPh}(\mathrm{OEt})_{2}(\mathrm{~b}), \mathrm{PPh}_{2} \mathrm{OEt}(\mathrm{c})\right]$. To a solution of $\mathrm{RuCl}_{2}(\mathrm{bpy})_{2} \cdot 2 \mathrm{H}_{2} \mathrm{O}(0.5 \mathrm{~g}, 0.96 \mathrm{mmol})$ in $15 \mathrm{~mL}$ of ethanol was added first an excess of the appropriate phosphite $(1.9 \mathrm{mmol})$ and then an excess of $\mathrm{NaBH}_{4}(0.36 \mathrm{~g}, 9.6 \mathrm{mmol})$ in $15 \mathrm{~mL}$ of ethanol. The reaction mixture was refluxed for $30 \mathrm{~min}$ and then the solvent was removed under reduced pressure. From the resulting oil the hydride complex $\left[\mathrm{RuH}(\mathrm{bpy})_{2} \mathrm{P}\right] \mathrm{Cl}$ was extracted with three 5-mL portions of dichloromethane and the extracts evaporated to dryness. An excess of $\mathrm{LiCF}_{3} \mathrm{SO}_{3}$ ( $4 \mathrm{mmol}, 0.62 \mathrm{~g}$ ) in $4 \mathrm{~mL}$ of ethanol was added to the oil obtained and the resulting solution was stirred for $1 \mathrm{~h}$. The solvent was removed under reduced pressure giving a red-brown oil from which the $\left[\mathrm{RuH}(\mathrm{bpy})_{2} \mathrm{P}\right] \mathrm{CF}_{3} \mathrm{SO}_{3}$ was extracted with three 5 -mL portions of diethyl ether. By cooling to $-25^{\circ} \mathrm{C}$ the extracts, red-brown microcrystals of the complex separated out, which was filtered and dried under vacuum; yield $\geq 20 \%$. Anal. Calcd for $\mathrm{C}_{27} \mathrm{H}_{32} \mathrm{~F}_{3} \mathrm{~N}_{4} \mathrm{O}_{6} \mathrm{PRuS}\left(\mathbf{8 a}-\mathbf{C F}_{3} \mathbf{S ~ O}_{3}\right)$ : C, 44.44; H, 4.42; N, 7.68. Found: C, 44.58; H, 4.29; N, 7.50. $\Lambda_{\mathrm{M}}=88.5 \Omega^{-1} \mathrm{~mol}^{-1} \mathrm{~cm}^{2}$. IR (KBr): $1890 \mathrm{~m}[\mathrm{v}(\mathrm{RuH})] \mathrm{cm}^{-1} .{ }^{1} \mathrm{H}$ NMR $\left[\mathrm{CD}_{2} \mathrm{Cl}_{2}, 25^{\circ} \mathrm{C} ; \delta\right]: 9.42-7.05$ (m, $8 \mathrm{H}$, bpy), 3.78 (qnt, 6 $\left.\mathrm{H}, \mathrm{CH}_{2}\right), 1.10\left(\mathrm{t}, 9 \mathrm{H}, \mathrm{CH}_{3}\right),-12.18\left(\mathrm{~d}, 1 \mathrm{H}, \mathrm{H}^{-}\right) .{ }^{31} \mathrm{P}\left\{{ }^{1} \mathrm{H}\right\} \mathrm{NMR}\left[\mathrm{CD}_{2} \mathrm{Cl}_{2}, 25^{\circ} \mathrm{C} ; \delta\right]: 153.0$ s. Anal. Calcd for $\mathrm{C}_{31} \mathrm{H}_{32} \mathrm{~F}_{3} \mathrm{~N}_{4} \mathrm{O}_{5} \mathrm{PRuS}\left(\mathbf{8 b}-\mathbf{C F}_{3} \mathbf{S O}_{3}\right.$ ): C, 48.88; H, 4.23; N, 7.36. Found: C, 48.70; H, 4.22; N, 7.30. $\Lambda_{\mathrm{M}}=75.5 \Omega^{-1} \mathrm{~mol}^{-1} \mathrm{~cm}^{2}$. IR $(\mathrm{KBr}): 1895 \mathrm{~m}[v(\mathrm{RuH})] \mathrm{cm}^{-1} .{ }^{1} \mathrm{H}$ NMR $\left[\mathrm{CD}_{2} \mathrm{Cl}_{2}\right.$, $25^{\circ} \mathrm{C}$; d]: 9.58-7.10 (m, $13 \mathrm{H}, \mathrm{Ph}$ and bpy), 3.96 (qnt, $4 \mathrm{H}, \mathrm{CH}_{2}$ ), 1.28 (t, $6 \mathrm{H}, \mathrm{CH}_{3}$ ), -12.04 (d, 1 $\left.\mathrm{H}, \mathrm{H}^{-}\right) .{ }^{31} \mathrm{P}\left\{{ }^{1} \mathrm{H}\right\} \mathrm{NMR}\left[\mathrm{CD}_{2} \mathrm{Cl}_{2}, 25{ }^{\circ} \mathrm{C} ; \delta\right]: 174.5$ s. Anal. Calcd for $\mathrm{C}_{35} \mathrm{H}_{32} \mathrm{~F}_{3} \mathrm{~N}_{4} \mathrm{O}_{4} \mathrm{PRuS}(8 \mathrm{c}-$ $\mathbf{C F}_{3} \mathrm{SO}_{3}$ ): C, 52.96; H, 4.06; N, 7.06. Found: C, 52.83; H, 4.12; N, 7.14. $\Lambda_{\mathrm{M}}=81.2 \Omega^{-1} \mathrm{~mol}^{-1}$ $\mathrm{cm}^{2}$. IR (KBr): $1910 \mathrm{~m}[v(\mathrm{RuH})] \mathrm{cm}^{-1} .{ }^{1} \mathrm{H}$ NMR $\left[\mathrm{CD}_{2} \mathrm{Cl}_{2}, 25^{\circ} \mathrm{C} ; \delta\right]: 9.45-7.12(\mathrm{~m}, 18 \mathrm{H}, \mathrm{Ph}$ and bpy), 3.70 (qnt, $\left.2 \mathrm{H}, \mathrm{CH}_{2}\right), 1.06\left(\mathrm{t}, 3 \mathrm{H}, \mathrm{CH}_{3}\right),-11.92\left(\mathrm{~d}, 1 \mathrm{H}, \mathrm{H}^{-}\right) .{ }^{31} \mathrm{P}\left\{{ }^{1} \mathrm{H}\right\} \mathrm{NMR}\left[\mathrm{CD}_{2} \mathrm{Cl}_{2}, 25{ }^{\circ} \mathrm{C}\right.$; $\delta]: 148.1 \mathrm{~s}$. 